\title{
Patterns of Maxillary Canine Impaction in Iraqi Population
}

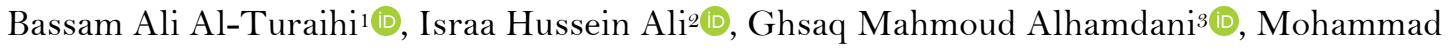 \\ Khursheed Alam ${ }^{4}$ (D)
}

\begin{abstract}
${ }^{1}$ Department of Orthodontics, College of Dentistry, University of Al-Kafeel, Al-Najaf, Iraq.
${ }^{2}$ Department of Pediatric and Preventive Dentistry, College of Dentistry, University of Babylon, Al-Hilla, Babil, Iraq. ${ }^{3}$ Department of Prosthodontics, College of Dentistry, University of Al-Kafeel, Al-Najaf, Iraq.

${ }^{4}$ Orthodontic Department, College of Dentistry, Jouf University, Sakaka, Aljouf, Kingdom of Saudi Arabia.
\end{abstract}

Author to whom correspondence should be addressed: Bassam Ali Al-Turaihi, Department of Orthodontics, College of Dentistry, University of Al-Kafeel, Al-Najaf, Iraq. Phone: +9647901674327. E-mail: bassam.alturaihi@alkafeel.edu.iq.

Academic Editor: Alessandro Leite Cavalcanti

Received: 27 October 2019 / Accepted: 19 May 2020 / Published: 26 June 2020

How to cite this article: Al-Turaihi BA, Ali IH, Alhamadani GM, Alam MK. Patterns of maxillary canine impaction in Iraqi population. Pesqui Bras Odontopediatria Clín Integr. 2020; 20:e5266. https://doi.org/10.1590/pboci.2020.120

\begin{abstract}
Objective: To investigate the prevalence of maxillary canine impaction and whether the impacted canine cases have had a potentially good or poor prognosis if interceptive treatment was practiced at earlier ages. Material and Methods: 1755 panoramic radiographs of patients, aged 13 to 60 years, attending the University of Babylon, College of Dentistry for the period from November 2016 to July 2018 were collected and assessed for the presence of impacted maxillary canines. Subsequently, four suggested prognostic factors for each impacted canine were analyzed and recorded. Results: The prevalence of maxillary canine impaction was $119(6.78 \%)$ with 89 unilateral impaction and 30 bilateral impaction resulting in a total of 149 impacted canines. Regarding the gender, impaction was more evident in females 70 than in males 49 with no statistical significant difference. For the side, impaction was more prevalent on the left (77) than on the right (72), also with no statistical difference $(\mathrm{p}>0.05)$. Following the analysis of the four prognostic factors, most of the cases, $117(78.5 \%)$ had at least one prognostic factor to be poor. Although $82(55 \%)$ of the cases had at least one prognostic factor to be good, only 4 had all the prognostic factors to be good. Conclusion: Although the cases that had a potentially good prognosis were little (4 cases), interceptive extraction of primary canines would mostly be beneficial for these patients as it will minimize the treatment time, cost and complications.
\end{abstract}

Keywords: Orthodontics, Interceptive; Tooth, Impacted; Radiography, Panoramic. 


\section{Introduction}

Canine is considered as the cornerstone of the upper arch. It plays an essential role in facial appearance, dental aesthetic, arch development, and functional occlusion [1]. Maxillary canine has the longest period of development and the most tortuous route to full occlusion. Therefore, it is considered as the second most common tooth to be impacted following third molars [1-4].

The prevalence of maxillary canine impaction is approximately $1-5$ percent [3]. This divergence is owed to different populations studied [2]. Maxillary canine's impaction occurs twice in females as in males, and about $8 \%$ of canine impaction is bilateral [2,3,5-7].

The tooth is considered to be impacted when it is restrained from erupting into the oral cavity within the time and physiological limits of the normal eruption process because of malposition, lack of space or other impediments $[1,3,8]$. Even prior to its eruption, impaction could also be considered when the tooth is expected to undergo abnormal emergence as a result of tooth germ location, tooth shape, eruption direction, and space available [3]. The average age when a maxillary canine should erupt is 13 years in boys and 12 years and 3 months in girls [8].

The ectopically positioned maxillary canine may diverge either buccally or palatally [9]. Palatal impaction was reported to be more than three times buccal impaction [2,7,10-12]. Malposed maxillary canine may also be in the line of the arch, or it could even lie horizontally above the apices of the maxillary incisors or displaced near the nose [7].

The main cause of buccal impaction of canine is inadequate arch space or crowding [13]. On the other hand, two theories have been proposed to explain palatal impaction of canine: the guidance theory and the genetic theory. The guidance theory proposed that the distal aspect of the lateral incisor is the guide for canine eruption. Therefore, the presence of spaces in spaced dentition, abnormally small mesio-distal width of the pegshaped lateral or congenitally missing lateral incisor may result in palatal impaction of canine. The genetic theory is based on the genetic cause [9]. It suggested that palatal impaction of canine is associated with other dental anomalies such as peg-shaped or missing lateral incisor, second premolar aplasia, infra-occlusion of primary molars [14]. Other factors include supernumerary teeth, odontomes, cystic lesions, delayed exfoliation of primary canine, early trauma to the maxilla, cleft lip and palate, ankylosis, crypt displacement, long eruption path, and syndromes such as Cleidocranial dysplasia [7,12].

An impacted canine assessment involves a clinical examination and radiographic investigation, which encompasses two-dimensional radiographs such as panoramic, periapical, cephalometric, lateral skull and maxillary occlusal views or the three dimensional Cone Beam Computerized Technology (CBCT) [7].

Clinical assessment usually involves visual inspection of the canine bulge and palpation using the index fingers of both hands simultaneously. As the maxillary canine erupts from 9.3 to 13.1 years, and it is usually palpable from 1 to 1.5 years before their emergence. Therefore, from the age of 8 , the buccal surface of the alveolar process distal to the lateral incisor should be palpated to detect the upper canine position. The absence of canine bulge after the age of 10 years strongly suggests that the tooth is displaced from its normal position. At this point, radiographic investigation will be done [5,7,12].

The two-dimensional radiographic technique was the standard method of choice for detecting maxillary canine impaction until recently. This is attributed to its relatively low emitted radiation dose as well as its availability in the standard dental office [5].

Impaction of maxillary canine may have a negative effect on the tooth itself as well as on the adjacent structures. Some of them are follicular cyst formation, internal resorption of the impacted canine, ankylosis, 
infection and tipping of adjacent teeth with loss of arch length and most commonly external root resorption of adjacent teeth $[3,5,7,8]$. The treatment options available for managing impacted canines involve no active treatment, i.e., leave in situ and monitor radiographically for cyst formation, interceptive treatment, surgical exposure and orthodontic alignment, surgical repositioning and extraction [7].

The delay in diagnosing the potentially impacted canine usually leads to more complicated treatment modalities such as surgical exposure and orthodontic alignment. This approach may cause destruction to the tooth's supporting structures (bone loss, root resorption, and gingival recession), but may also require long treatment duration and be costly to the patient [15]. Therefore, early diagnosis and interceptive management are paramount to minimize treatment time, complexity, complications, and costs $[5,8,12,16]$.

The preventive treatment of choice for impacted canine in class I non-crowded cases involves extraction of primary canine at the age of 10-13 years [7,12,13]. This approach may be conjugated by using cervical pull headgear [17], or rapid maxillary expander [8,10].

This interceptive management has supported the theory that the presence of primary canine would become a mechanical obstacle for the eruption of the permanent canine [8]. This was confirmed by a previous study, which found that $78 \%$ of impacted canines in 10-13 years-old children have spontaneously erupted within 6-12 months following extraction of their primary predecessors [18].

An estimation for the efficiency of interceptive treatment can be carried out by assessing four prognostic factors [7]. These factors involve the amount of canine crown horizontally overlaps the adjacent incisor, the vertical height of the canine crown, canine angulation to the midline, and the position of the canine root apex in the horizontal plane. For all of these prognostic factors, the prognosis can range from good to average to poor depending on the position of the impacted canine to the adjacent structures [7].

These four criteria may aid decision making for management of impacted maxillary canines. If canine prognosis in all four categories is good, then the decision for extracting primary canine will mostly allow spontaneous eruption of impacted canine. Similarly, when the prognosis is average, management usually involves extraction of primary canine. If the tooth did not erupt within a year, orthodontic treatment would be indicated, which involves exposure and alignment. Lastly, if one or more of the criteria suggest a poor prognosis, orthodontic treatment will be necessary and primary canine should not be extracted [7].

As an analysis of these four criteria can assist the dental clinician in anticipating the potential outcome for the impacted maxillary canine following interceptive treatment, and as the panoramic radiograph can aid in recording these criteria, the aim of this study was firstly to report the prevalence of maxillary canine impaction in a sample of Iraqi population aged 13 to 60 years and secondly to assess whether the impacted canine cases have had a potentially good or poor prognosis if interceptive extraction of primary canines was practiced at earlier stages.

\section{Material and Methods}

Study Design and Sample

This is a retrospective descriptive study that assesses all the panoramic radiographs of patients attending the University of Babylon, College of Dentistry, from November 2016 to July 2018.

The panoramic radiographs were taken for different purposes, including assessment of developing dentition, oral surgical procedures, investigating oral pathology, and orthodontic diagnosis. The total number of the panoramic radiographs gathered was 2193 radiographs; however, only 1755 radiographs were included. 
The maxillary canine was considered to be impacted when it was obstructed on its path of eruption by an adjacent tooth, bone, or soft tissue from the age of 13 years. As the permanent maxillary canine is usually erupting at the age of 12 to 13 years, the inclusion criteria for this study involved inspecting the radiographs of patients aged 13 to 60 years old [19-22].

Following the inspection of the panoramic radiographs, patients who displayed one or more of the following pathological conditions were excluded from the study [1,3]: Any evident anomaly such as odontomas or supernumerary teeth; Any trauma or fracture of the jaw that might affect the normal development of permanent teeth; and the presence of multiple impacted teeth which may imply the presence of a syndrome.

\section{Data Collection}

The Orthopantomograph GXDP-700 (Gendex Dental Systems, Hatfield, USA) was used for the panoramic radiographs collected for the present study set at 1.25 magnification, as suggested by the manufacturer.

All radiographs were acquired with a standardized head position and stabilized by head rods. All digital images were stored in a computer database using the manufacturer's software (VixWin Platinum, Gendex, KaVo Dental, Brea, CA, USA). A specialist Orthodontist examined all the panoramic radiographs. Each image was magnified to $110 \%$ and contrast and brightness were optimized to produce the best image for viewing under standardized conditions. The panoramic radiographs that satisfied the criteria as mentioned were 119 radiographs, with a total of 149 impacted canines. Of these, thirty radiographs had bilateral canine impaction.

The study variables involved the patient's name, age, gender and the date at which the radiograph was taken. The primary outcome variable was the presence of impacted maxillary canine, either unilaterally or bilaterally. The other variable was the position of the impacted canine(s), which was analyzed using four factors [7] (Table 1). The prognosis for each factor was arranged in ascending order from 1 to 3. This ascending prognosis is illustrated in Table 2 from some of the examined radiographs.

Table 1. The four prognostic factors assessed for the impacted maxillary canines [7].

\begin{tabular}{|c|c|c|}
\hline Prognostic Factor & Criteria & $\begin{array}{c}\text { Level of Prognosis } \\
\text { Following Interceptive } \\
\text { Treatment } \\
\end{array}$ \\
\hline \multirow{3}{*}{$\begin{array}{l}\text { 1. The amount of canine crown } \\
\text { horizontally overlaps the adjacent } \\
\text { incisor }\end{array}$} & No horizontal overlap & Good Prognosis \\
\hline & Overlap up to half root width & Average Prognosis \\
\hline & Complete overlap & Poor Prognosis \\
\hline \multirow[t]{3}{*}{ 2. Vertical height of the canine crown } & $\begin{array}{l}\text { Cemento-enamel junction of impacted canine lies } \\
\text { halfway up to the root of adjacent incisor }\end{array}$ & Good Prognosis \\
\hline & Cemento-enamel junction $>$ halfway $<$ full root length & Average Prognosis \\
\hline & Cemento-enamel junction $>$ full root length & Poor Prognosis \\
\hline \multirow[t]{3}{*}{ 3. Canine angulation to the midline } & Angulation of $0-15^{\circ}$ & Good Prognosis \\
\hline & Angulation of $16-30^{\circ}$ & Average Prognosis \\
\hline & Angulation of $31^{\circ}$ or more & Poor Prognosis \\
\hline \multirow{3}{*}{$\begin{array}{l}\text { 4. The position of the canine root } \\
\text { apex in the horizontal plane }\end{array}$} & Above canine position & Good Prognosis \\
\hline & Above first premolar & Average Prognosis \\
\hline & Above second premolar & Poor prognosis \\
\hline
\end{tabular}


Table 2. The levels of prognosis for the prognostic factors for some of the examined radiographs [7].

\begin{tabular}{|c|c|c|c|}
\hline Prognostic Factor/ Prognosis & 1. Good & 2. Average & 3. Poor \\
\hline $\begin{array}{l}\text { 1. The amount of canine crown } \\
\text { horizontally overlaps the } \\
\text { adjacent incisor }\end{array}$ & & & \\
\hline $\begin{array}{l}\text { 2. Vertical height of the canine } \\
\text { crown }\end{array}$ & & & \\
\hline $\begin{array}{l}\text { 3. Canine angulation to the } \\
\text { midline }\end{array}$ & & & \\
\hline $\begin{array}{l}\text { 4. The position of the canine } \\
\text { root apex in the horizontal } \\
\text { plane }\end{array}$ & & & \\
\hline
\end{tabular}

For unilateral impaction cases, one set of all four prognostic factors was recorded, and for the bilateral impaction cases, two sets were registered.

To test the maxillary canine impaction assessment's reliability, $10 \%(\mathrm{n}=175)$ panoramic radiographs were selected randomly and re-inspected by a specialist in pediatric dentistry. Kappa test was used to measure the level of agreement between the two observers. There was no significant inter-rater difference $(p>0.05)$ for the $10 \%$ of the radiographs selected to assess reliability. The level of agreement between the two observers was excellent $($ Kappa $=0.89)$.

Data Analysis

For statistical analysis, data were gathered and analyzed using Microsoft Excel spreadsheet 2010 and the SPSS statistical package (version 23) (SPSS Inc., Chicago, Illinois, USA). The differences between gender, age and side of impaction were tested using the Chi-square test. Whereas statistical comparisons with the four 
prognostic factors were made using the one-way analysis of variance test (ANOVA). For all analyses, a $\mathrm{P}$ value of less than 0.05 was considered to be statistically significant.

\section{Results}

Out of the 1755 radiographs included in this study, 788 (44.9\%) were for males and $967(55.1 \%)$ were for females (Table 3). The age of the patients included ranged from 13 to 60 years, with a mean of 26 years. The sample was divided into five subgroups, according to the selected age range, with group1) 13-20 years $(\mathrm{n}=514)$; group 2) 21-30 years $(\mathrm{n}=813)$; group 3) 31-40 years $(\mathrm{n}=296)$; group 4) 41-50 years $(\mathrm{n}=86)$; and group 5) 51-60 years $(\mathrm{n}=46)$ (Table 4$)$.

Following examining the included 1755 radiographs, maxillary canine impaction was detected on 119 $(6.78 \%)$ radiographs (Table 3$)$. Of these, 89 cases had unilateral maxillary canine impaction and thirty cases had bilateral impaction, resulting in a total of 149 impacted maxillary canines. Maxillary canine impaction was more in females $(n=70)$ than in males $(n=49)$. The percentage of canine impaction for each gender was also more in females $(7.2 \%)$ than in males $(6.2 \%)$ with no statistical significant difference (p>0.05) (Table 3). Likewise, impacted maxillary canine was more prevalent on the left-hand side $(n=77)$ than on the right $(n=72)$; however, this difference was not significant statistically $(\mathrm{p}>0.05)$.

Table 3. Distribution of subjects according to gender.

\begin{tabular}{lccc}
\multicolumn{1}{c}{ Gender } & $\mathbf{N}(\%)$ & \multicolumn{2}{c}{ Impacted Canine Cases } \\
& & N & $\%$ \\
\hline Male & $788(44.9)$ & $49(41.2)$ & 6.2 \\
Female & $967(55.1)$ & $70(58.8)$ & 7.2 \\
Total & $1755(100.0)$ & $119(100.0)$ & 6.8 \\
\hline
\end{tabular}

For the subgroups divided, approximately half of the total impacted cases (60 subjects) were for group 1. Group 2 had 45 cases with impacted maxillary canine (37.8\%), and group 3 had 13 cases $(10.9 \%)$. On the other hand, group 4 had no detected maxillary canine impaction cases, and group 5 had only one case of maxillary canine impaction, which represents less than $1 \%$ of the total impacted cases. This gives group 1 the highest prevalence for maxillary canine impaction $(11.6 \%)$ that was significantly different from the other groups $(\mathrm{p}<0.05)($ Table 4$)$.

Table 4. Distribution of subjects according to age groups.

\begin{tabular}{ccccc}
\hline Group & Age Range & N & Impacted Canine Cases \\
& & & N & $\%$ \\
\hline 1 & $13-20$ Years & 514 & 60 & 11.6 \\
2 & $21-30$ Years & 813 & 45 & 5.5 \\
3 & $31-40$ Years & 296 & 13 & 4.3 \\
4 & $41-50$ Years & 86 & 0 & 0.0 \\
5 & $51-60$ Years & 46 & 1 & 2.2 \\
Total & & 1755 & 119 & 6.78 \\
\hline
\end{tabular}

The radiographs that included impacted maxillary canines had undergone further analysis to detect the likely prognosis following early detection and early interceptive intervention. Four prognostic factors were analyzed once for the unilateral impaction, while for the thirty cases of bilateral impaction, double analysis was performed for each impacted canine. Therefore, a total of 149 impacted canine were assessed. 
For the first prognostic factor, the amount of canine crown horizontally overlaps the adjacent incisor, 35 (23.5\%) impacted canine crowns had no horizontal overlap, 17 (11.4\%) crowns had overlapped up to the half of the adjacent incisor's root, and $97(65.1 \%)$ of the impacted canines had completely overlapped the neighboring incisor's root horizontally.

The second prognostic factor, the vertical height of the canine crown; 64 (43\%) impacted canine crowns had their CEJ lies halfway up to the root of the adjacent incisor, 71 (47.6\%) impacted canine crowns had their CEJ more than halfway but less than full length of the adjacent incisor root. Only 14 (9.4\%) of the impacted canine crowns lies up in the upper jaw with their CEJ located more than full root length of the neighboring incisor.

For the canine angulation to the midline factor, 13 (8.7\%) impacted canines had an angulation of 0-15, $40(26.9 \%)$ of impacted canines had an angulation of 16-30, and 96 (64.4\%) impacted canines had an angulation of 31 or more.

Finally, The horizontal position of the canine root apex. 16 (10.7\%) impacted canine apices located above canine position, 89 (59.7\%) of impacted canine apices positioned above the first premolar, and 44 (29.6\%) impacted canine apices situated above the second premolar (Table 5).

Table 5. Distribution of impacted canines according to the four prognostic factors.

\begin{tabular}{lccccc}
\multicolumn{1}{c}{ Prognostic Factor } & \multicolumn{4}{c}{ The Level of Prognosis } \\
& 1 & 2 & 3 & Total \\
& $\mathrm{N}(\%)$ & $\mathrm{N}(\%)$ & $\mathrm{N}(\%)$ & $\mathrm{N}(\%)$ \\
\hline Amount of Canine Crown Horizontally Overlaps the Adjacent Incisor & $35(23.5)$ & $17(11.4)$ & $97(65.1)$ & $149(100.0)$ \\
Vertical Height of Canine Crown & $64(43.0)$ & $71(47.6)$ & $14(9.4)$ & $149(100.0)$ \\
Canine Angulation to the Midline & $13(8.7)$ & $40(26.9)$ & $46(64.4)$ & $149(100.0)$ \\
Horizontal Position of Canine Root Apex & $16(10.7)$ & $89(59.7)$ & $44(29.6)$ & $149(100.0)$ \\
\hline
\end{tabular}

Only three cases of impacted maxillary canines had a good prognosis for all the four prognostic factors. The age of these patients was 13, 16, and 17 years old patient with bilateral impaction. These patients would benefit from interceptive treatment at an early age (Figure 1). On the other hand, three cases had all four prognostic factors to be poor. The age of these patients was 16, 20, and 35 years-old. These patients would not benefit from extraction of their primary canine (Figure 2).

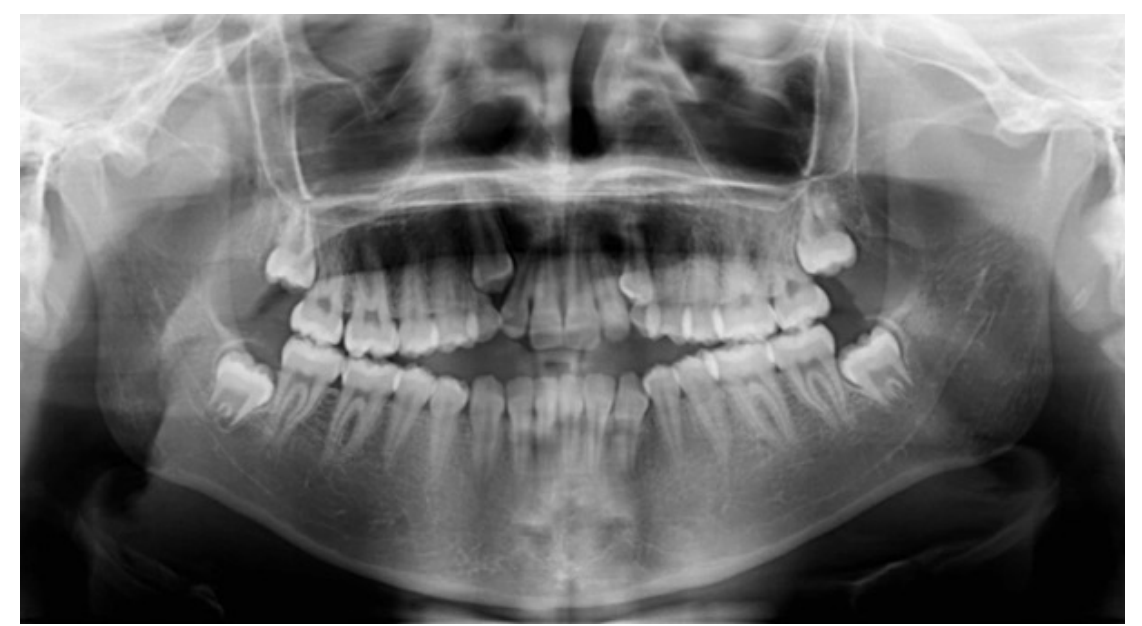

Figure 1. A panoramic radiograph of 16 years-old patient with bilateral maxillary canine impaction with the right canine has three good prognostic factors and the left canine. 


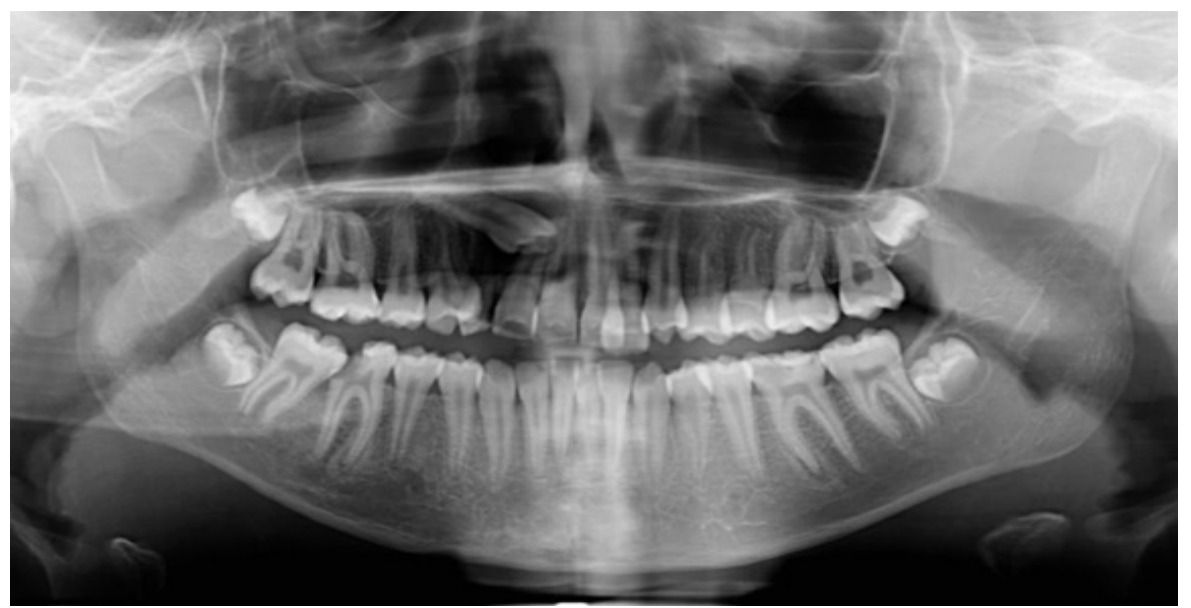

Figure 2. A panoramic radiograph of 16 years-old patient with unilateral maxillary canine impaction with the right impacted canine has all the prognostic factors to be poor.

Most of the cases $(n=117)$ had at least one prognostic factor to be poor. This represents $78.5 \%$ of the whole 149 impacted canines. Similarly, the average prognosis was also high, with 133 subjects (89.3\%) having at least one prognostic factor with an average prognosis. However, no cases were detected to have all the four prognostic factors to be average.

The percentage of poor prognosis was the highest for the amount of canine crown horizontally overlaps the adjacent incisor (factor 1) and also for the canine angulation to the midline (factor 3 ) (65.1\% and $64.4 \%$ respectively). On the other hand, the percentage of the average prognosis was the highest for the vertical height of the canine crown (factor 2 ) and for the horizontal position of the canine root apex (factor 4 ) (47.6\% and $59.7 \%$ respectively). This leaves the percentage of good prognosis to be the lowest for factors 3 and 4; and the second-lowest for prognostic factors 1 and 2 (Figure 3). The statistical analysis has shown no significant difference among the four prognostic factors concerning gender, age, or side of impaction ( $\mathrm{p}>0.05)$.

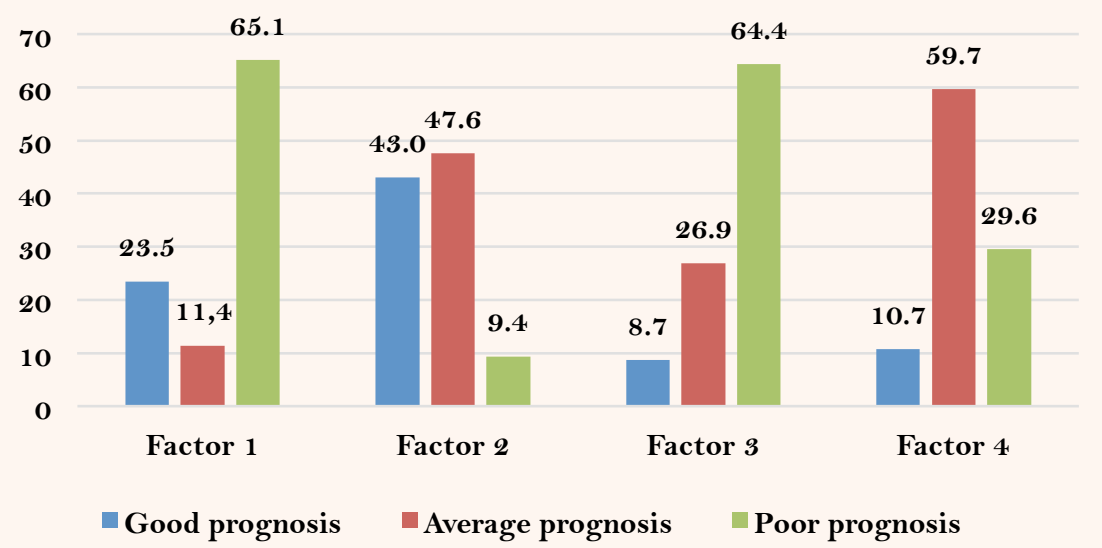

Figure 3. Percentage distribution of prognostic factors.

\section{Discussion}

Management of impacted maxillary canine continues to be a frustrating practice for the multidisciplinary clinicians treating the $2 \%$ of orthodontic patients having this pathology [8]. Treatment options vary with the age of the patient at diagnosis, degree of an impaction as well as the canine position [7]. 
Delayed diagnosis often requires surgical exposure of the impacted canine followed by orthodontic alignment to direct the tooth into the normal position within the dental arch [8]. This procedure is not only costly and requires long treatment time, but may also be associated with undesirable disruption to the supporting tooth structures such as bone loss, root resorption and gingival recession [15]. Therefore, early diagnosis and intervention were suggested by many authors to reduce treatment complexity, expenses, time and potential complications $[8,12,13,16-18]$.

In this study, 2193 panoramic radiographs of patients attending the University of Babylon, College of Dentistry for the period from November 2016 until July 2018 were investigated. One thousand seven hundred fifty-five panoramic radiographs for patients aged 13 to 60 years-old were included in the study. Although, it has been found by one study [23] that impaction of maxillary canine can be detected on a single panoramic radiograph as early as 8 years, and as panoramic radiographs are indicated for 10 years-old patients who do not have canine bulge or those who have missing lateral incisor, peg-shaped lateral incisor or over-retained primary canine $[5,7,12,13]$, the age group selected for the present study starts from the age of 13 years and extended to the age of 60 years. This age group was selected because within this range, usually all permanent teeth are erupted and the impacted maxillary canine can be detected reliably. Also, previous studies had used patients older than 13 years of age as their experimental material to detect impacted maxillary canines [19$21,24,25]$.

Following early detection of impacted maxillary canine, interceptive treatment that involves extraction of the primary canines must commence as early as 10 years up to 13 years. This approach is applicable for class I non-crowded cases and when most of the four prognostic factors suggest good prognosis for the impacted canine [7]. Once more, the age group chosen for this study was higher than the age range suggested beginning interceptive treatment. The authors intended to investigate whether the cases of impacted maxillary canines with older ages and good prognosis for the four prognostic factors would benefit from the early intervention if they were detected on the panoramic radiographs at earlier time.

Although Cone Beam Computed Tomography (CBCT) has been shown to be more sensitive and significantly better than the conventional panoramic radiograph in detecting the position of impacted maxillary canine [6] as well as identifying the external root resorption of the adjacent lateral incisor [26], the twodimensional panoramic radiographs were used in this study to assess the presence of impacted maxillary canine as well as the pattern of impaction rather than the contemporary three-dimensional CBCT. The justification was based on the fact that a single panoramic radiograph can be used reliably to detect the position of the impacted maxillary canine with high specificity and sensitivity [2]

Also, the current UK and European guidance have suggested using the small FOV CBCT for assessing impacted canine only in selected cases where conventional radiographs fail to provide sufficient information to construct a treatment plan. Furthermore, the British Orthodontic Society - Orthodontic Radiographs guidelines have reported that there is no indication for the routine use of CBCT to detect impacted maxillary canines as the CBCT technique is associated with a higher overall effective dose of ionizing radiation than the conventional radiography [16]. Moreover, CBCT is usually used to overcome the problem of underestimating the degree of root resorption of the adjacent teeth on a plain radiograph [7], which was not investigated in the present study. For all of these reasons, the panoramic radiograph was chosen as the experimental material for this study. 
For the first aim of this study, the prevalence of impacted maxillary canine. The detected prevalence was $(6.78 \%)$. This prevalence was higher than the prevalence reported in the literature (1-5\%) [1]. It was also higher than the prevalence found in Iraqi population (2.7\%) [20], in Turkish population (1.74\%) [19], in Western Saudi population (3.3\%) [27], in Cypriote population (3.53\%) [24] and in Croatian population (4.71\%) [25]. However, the present study prevalence was less than the prevalence detected in North Greek population (8.4\%) [28], and in the North Indian population (9.7\%) [22]. This variation in reporting the prevalence may firstly be due to the difference in the population studied [8], or it could secondly be due to the type of patients studied, as most of those patients who required a panoramic radiograph were looking for orthodontic treatment.

Regarding the gender of the studied sample, the number of impacted canines were higher in females than in males; however, this number was not as high as the twice ratio reported previously [3,6-8]. The difference was also not statistically significant as that reported in the Iraqi population study [20], and the North Indian population study [22] For the side of impaction, impaction was more evident on the left-hand side than on the right; however, this difference was not significant statistically, which was similar to that reported by other authors [20,24]. Despite being also not statistically different in other studies [2,3,19], canine impaction on the right side was more prevalent.

When the studied cases categorized into five subgroups, group 2 (21-30 years), group 3 (31-40 years) and group 5 (51-60) had a prevalence of 5.5\%, 4.3\%, and $2.2 \%$ respectively, which was coincident with the prevalence reported in the literature. On the other hand, group 1 (13-20 years) had a prevalence of $11.6 \%$ that was as high as the prevalence reported for patients with Down Syndrome (12\%) [29].

The second objective of the present study was to assess the probable prognosis of the impacted cases if detected and managed at an earlier age. For this aim, four prognostic factors were used, as proposed previously [7]. No previous published studies have fully investigated these four prognostic factors; however, some of these factors were previously utilized by authors intending to determine the position of the impacted maxillary canine as precisely as possible. The number of canine crown horizontally overlaps the adjacent incisor factor used by the studies $[3,15,21,23]$. The vertical height of the canine crown factor was also employed by other authors [21], as well as the canine angulation to the midline factor [15,23]. This angle was also compared in previous research [30] with another three methods used different angles to assess the reliability of locating the inclination of impacted maxillary canines. This study's results have shown that this angle is a reliable indicator used on the panoramic radiographs to detect the position of the impacted maxillary canine compared to the other three methods.

The first and third prognostic factors have shown a high percentage of poor prognosis $(65.1 \%$ and $64.4 \%$, respectively). In contrast, the second and fourth prognostic factors demonstrated a high percentage of average prognosis $(47.6 \%$ and $59.7 \%$, respectively). For all of the four prognostic factors analyzed, the good prognosis represented the lowest or the second-lowest percentage. This indicates a high percentage of poor prognosis for most of the collected cases.

When categorizing the samples collected according to age groups, approximately $50 \%$ of the impacted cases $(n=60)$ were identified in group 1 (13 to 20 years). Only three cases $(2.5 \%)$ within this group had all the four prognostic factors to be good. This suggests that those patients would benefit from interceptive intervention at the age of 10 to 13 by extracting the primary canines and allow the impacted maxillary canines to align spontaneously and erupt eventually as recommended by some authors [7,18]. The average prognosis was detected in 17 cases (28\%). Those patients may also benefit from early extraction of their primary 
predecessors; yet, their impacted maxillary canine must be monitored for spontaneous eruption within a year as failure to emerge to the oral cavity within this time limit will suggest active orthodontic treatment.

For the 59 subjects with impacted maxillary canine aged older than 20 years (groups 2, 3 and 5), 93\% of those cases $(n=55)$ had at least one prognostic factor to be poor. This indicates that those patients would not benefit from the interceptive extraction of the primary canines at an earlier age. However, orthodontic treatment with surgical exposure and active orthodontic traction is a substantial requirement for those who seek orthodontic management. The other 4 cases of these groups $(7 \%)$ had average prognosis and may also benefit from the early interceptive treatment and the one-year follow-up.

\section{Conclusion}

Although the impacted canine cases with potentially good prognosis were few, early detection with clinical examination and when required, radiographic evaluation for these patients is pivotal to intervene and extract the primary canines. This will allow the potentially impacted canines to spontaneously emerge into the oral cavity with minimum time, cost and complications.

\section{Authors' Contributions}

\begin{tabular}{|c|c|c|}
\hline BAA & (iD) $0000-0002-8635-3100$ & Conceptualization, Methodology and Formal Analysis. \\
\hline IHA & (iD) $0000-0002-5796-7365$ & Project Administration and Writing - Original Draft Preparation. \\
\hline GMA & (iD) $0000-0003-0157-5677$ & Investigation, Validation and Writing - Original Draft Preparation. \\
\hline MKA & (iD) $0000-0001-7131-1752$ & Supervision and Formal Analysis. \\
\hline
\end{tabular}

\section{Financial Support}

None.

\section{Conflict of Interest}

The authors declare no conflicts of interest.

\section{References}

[1] Watted N, Abu-Hussein M. Prevalence of impacted canines in arab population in Israel. Int J Public Health Res 2014; $2(6): 71-7$

[2] Katsnelson A, Flick WG, Susarla S, Tartakovsky JV, Miloro M. Use of panoramic x-ray to determine position of impacted maxillary canines. J Oral Maxillofac Surg 2010; 68(5):996-1000. https://doi.org/10.1016/j.joms.2009.09.022

[3] Kim Y, Hyun HK, Jang KT. The position of maxillary canine impactions and the influenced factors to adjacent root resorption in the Korean population. Eur J Orthod 2012; 34(3):302-6. https://doi.org/10.1093/ejo/cjroo2

[4] Lai CS, Bornstein MM, Mock L, Heuberger BM, Dietrich T, Katsaros C. Impacted maxillary canines and root resorptions of neighbouring teeth: a radiographic analysis using cone-beam computed tomography. Eur J Orthod 2012; 35(4):529-38. https://doi.org/10.1093/ejo/cjs037

[5] Alqerban A, Jacobs R, Lambrechts P, Loozen G, Willems G. Root resorption of the maxillary lateral incisor caused by impacted canine: a literature review. Clin Oral Investig 2009; 13(3):247-55. https://doi.org/10.1007/s00784-009-0262-8

[6] Alqerban A, Jacobs R, Fieuws S, Willems G. Comparison of two cone beam computed tomographic systems versus panoramic imaging for localization of impacted maxillary canines and detection of root resorption. Eur J Orthod 2011; 33(1):93-102. https://doi.org/10.1093/ejo/cjq034

[7] Counihan K, Al-Awadhi EA, Butler J. Guidelines for the assessment of the impacted maxillary canine. Dent Update 2013; 4O(9):770-7. https://doi.org/10.12968/denu.2013.40.9.770 
[8] Litsas G, Acar A. A review of early displaced maxillary canines: etiology, diagnosis and interceptive treatment. Open Dent J 2011; 5:39-47. https://doi.org/10.2174/1874210601105010039

[9] Peck S, Peck L, Kataja M. The palatally displaced canine as a dental anomaly of genetic origin. Angle Orthod 1994; 64(4):249-56.

[10] Baccetti T, Mucedero M, Leonardi M, Cozza P. Interceptive treatment of palatal impaction of maxillary canines with rapid maxillary expansion: a randomized clinical trial. Am J Orthod Dentofacial Orthop 2009; 136(5):657-61. https://doi.org/10.1016/j.ajodo.2008.03.019

[11] D’Amico RM, Bjerklin K, Kurol J, Falahat B. Long-term results of orthodontic treatment of impacted maxillary canines. Angle Orthod 2003; 73(3):231-8.

[12] Richardson G, Russell KA. A review of impacted permanent maxillary cuspids - diagnosis and prevention. J Can Dent Assoc 2000; 66(9):497-501.

[13] Jacobs SG. The impacted maxillary canine. Further observations on aetiology, radiographic localization, prevention/interception of impaction, and when to suspect impaction. Aust Dent J 1996; 41(5):310-6. https://doi.org/10.1111/j.1834-7819.1996.tbo3139.x

[14] Mercuria E, Cassetta M, Cavallini C, Vicari D, Leonardi R, Barbato E. Dental anomalies and clinical features in patients with maxillary canine impaction. Angle Orthod 2013; 83(1):22-8. https://doi.org/10.2319/02 1712-149.1

[15] Zuccati G, Ghobadlu J, Nieri M, Clauser C. Factors associated with the duration of forced eruption of impacted maxillary canines: a retrospective study. Am J Orthod Dentofacial Orthop 2006; 130(3):349-56. https://doi.org/10.1016/j.ajodo.2004.12.028

[16] Husain J, Burden D, McSherry P. Management of the palatally ectopic maxillary canine. Available from: https://www.rcseng.ac.uk/-/media/files/rcs/fds/publications/canine-guideline-2016.pdf. [Accessed January 14, 2019].

[17] Baccetti T, Leonardi M, Armi P. A randomized clinical study of two interceptive approaches to palatally displaced canines. Eur J Orthod 2008; 30(4):381-5. https://doi.org/10.1093/ejo/cjno23

[18] Ericson S, Kurol J. Early treatment of palatally erupting maxillary canines by extraction of the primary canines. Eur J Orthod 1988; 10(4):283-95. https://doi.org/10.1093/ejo/10.4.283

[19] Aktan AM, Kara S, Akgünlü F, Malkoç S. The incidence of canine transmigration and tooth impaction in Turkish population. Eur J Orthod 2010; 32(5):575-81. https://doi.org/10.1093/ejo/cjp151

[20] Lazim AI. The prevalence of impacted maxillary canine among iraqi patients of Al-Basrah city. J Baghdad Coll Dent 2016; 28(1):73-7.

[21] Nagpal A, Pai KM, Setty S, Sharma G. Localization of impacted maxillary canines using panoramic radiography. J Oral Sci 2009; 51(1):37-45. https://doi.org/10.2334/josnusd.51.37

[22] Patil S, Maheshwari S. Prevalence of impacted and supernumerary teeth in the North Indian population. J Clin Exp Dent 2014; 6(2):e1 16-20. https://doi.org/10.4317/jced.51284

[23] Sajnani AK, King NM. Early prediction of maxillary canine impaction from panoramic radiographs. Am J Orthod Dentofacial Orthop 2012; 142(1):45-51. https://doi.org/10.1016/j.ajodo.2012.02.021

[24] Kamiloglu B, Kelahmet U. Prevalence of impacted and transmigrate canine teeth in a Cypriote orthodontic population in the Northern Cyprus area. BMC Res Notes. 2014; 7:346. https://doi.org/10.1186/1756-0500-7-346

[25] Prskalo K, Zjača K, Škarić-Jurić T, Nikolić I, Anić-Miloševic S, Lauc T. The prevalence of lateral incisor hypodontia and canine impaction in Croatian population. Coll Antropol 2008; 32(4):1105-9.

[26] Alqerban A, Jacobs R, Souza PC, Willems G. In-vitro comparison of 2 cone-beam computed tomography systems and panoramic imaging for detecting simulated canine impaction-induced external root resorption in maxillary lateral incisors. Am J Orthod Dentofacial Orthop 2009; 136(6):764.e1-764.e1 1. https://doi.org/10.1016/j.ajodo.2009.03.036

[27] Afify AR, Zawawi KH. The Prevalence of dental anomalies in the western region of Saudi Arabia. ISRN Dent 2012; 2012:837270. https://doi.org/10.5402/2012/837270

[28] Fardi A, Kondylidou-Sidira A, Bachour Z, Parisis N, Tsirlis A. Incidence of impacted and supernumerary teeth - a radiographic study in a North Greek population. Med Oral Patol Oral Cir Bucal 2011; 16(1):e56-61. https://doi.org/10.4317/medoral.16.e56

[29] Shapira J, Chaushu S, Becker A. Prevalence of tooth transposition, third molar agenesis, and maxillary canine impaction in individuals with down syndrome. Angle Orthod 2000; 70(4):290-6.

[30] Parenti SI, Gatto MR, Gracco A, Bonetti GA. Reliability of different methods for measuring the inclination of the maxillary canines on panoramic radiographs. Orthod Craniofac Res 2013; 16(3):177-84. https://doi.org/10.1111/ocr.12020 